\title{
Impact of Pleural Effusion on Outcomes of Patients Receiving Osimertinib for NSCLC Harboring EGFR T790M
}

\author{
KENTARO MASUHIRO, TAKAYUKI SHIROYAMA, HIDEKAZU SUZUKI, SO TAKATA, \\ SHINGO NASU, HIROMUNE TAKADA, SATOMU MORITA, AYAKO TANAKA, \\ NAOKO MORISHITA, NORIO OKAMOTO and TOMONORI HIRASHIMA \\ Department of Thoracic Oncology, Osaka Habikino Medical Center, Habikino, Japan
}

\begin{abstract}
Background/Aim: Osimertinib has demonstrated promising efficacy in patients with epidermal growth factor receptor (EGFR) T790M-positive non-small cell lung cancer (NSCLC). We investigated the efficacy of osimertinib in such patients presenting with pleural effusion, which has been unclear to date. Patients and Methods: The medical records of all patients treated with osimertinib for advanced NSCLC with EGFR T790M between April 2016 and July 2017 at our Institution were retrospectively reviewed. Time to treatment failure (TTF) and overall survival (OS) were determined as endpoints. Results: Twenty-three patients (seven with pleural effusions) were treated with osimertinib. Patients with pleural effusion had significantly shorter median TTF than those without (3.7 vs. 12.8 months, respectively, $p=0.021$ ), as well as shorter median OS (7.8 months vs. not attained, respectively, $p=0.002)$. Metastasis to the brain, bone, and liver did not significantly influence our endpoints. Conclusion: Osimertinib monotherapy is less effective in patients with NSCLC with pleural effusions.
\end{abstract}

Osimertinib is a third-generation irreversible epidermal growth factor receptor (EGFR) tyrosine kinase inhibitor (TKI). The AURA3 trial showed that osimertinib improved progression-free survival (PFS) over first-line chemotherapy in patients with EGFR T790M-positive advanced non-small cell lung cancer (NSCLC) whose disease had progressed after EGFR-TKI therapy (1). Additionally, the recent FLAURA trial found that osimertinib led to significantly improved PFS over the first-generation EGFR-TKIs in

Correspondence to: Takayuki Shiroyama, MD, Department of Thoracic Oncology, Osaka Habikino Medical Center, 3-7-1, Habikino, Osaka, 583-8588, Japan. Tel: +81 729572121, Fax: +81 729583291, e-mail: takayuki.s12@hotmail.co.jp

Key Words: Osimertinib, pleural effusion, EGFR T790M, non-small cell lung cancer, metastases. treatment-naïve patients with EGFR mutation-positive advanced NSCLC (2). As such, osimertinib is expected to exhibit more benefits as it is further explored.

Previous studies found that the presence of EGFR mutation is significantly associated with pleural effusion $(3,4)$, and that the efficacies of first-or second-generation EGFR-TKIs are limited for patients with pleural effusion $(5,6)$. However, the efficacy of osimertinib in patients with EGFR T790Mpositive NSCLC with pleural effusion remains unclear.

Therefore, this retrospective analysis was conducted to investigate the efficacy of osimertinib in patients with $E G F R$ T790M-positive advanced NSCLC with pleural effusion at presentation.

\section{Patients and Methods}

Patients. The data for consecutive NSCLC patients harboring the EGFR T790M mutation who were treated with osimertinib at Osaka Habikino Medical Center between April 2016 and July 2017 were retrospectively examined. The Institutional Review Board at the Osaka Habikino Medical Center approved this study (approval number: 890). Data were obtained from electronic medical records, and the requirement for written informed consent was waived by the Institutional Review Board as this was a retrospective analysis of anonymized clinical data. The research was conducted in accordance with the 1964 Declaration of Helsinki and its later amendments.

Data collection and evaluation. Patients were initially administered oral osimertinib at $80 \mathrm{mg} /$ day; this dose was reduced to $80 \mathrm{mg} /$ day every other day or to $40 \mathrm{mg} /$ day upon the appearance of unacceptable toxicity, and treatment was discontinued if toxicities persisted thereafter. Osimertinib therapy was continued until unacceptable adverse events or remarkable disease progression (PD), defined as PD with a decline in performance status. The endpoints measured in this study were the time to treatment failure (TTF) and overall survival (OS). TTF was defined as the interval between the start of osimertinib therapy and treatment discontinuation for any reason, including disease progression, unacceptable toxicity and death. OS was calculated from the start of osimertinib therapy to the date of death. The treatment response 
Table I. Patient characteristics at baseline ( $n=23)$.

\begin{tabular}{|c|c|c|c|c|}
\hline & All $(n=23)$ & With pleural effusion $(n=7)$ & Without pleural effusion $(n=16)$ & $p$-Value \\
\hline Median age (range), years & $73(68-77)$ & $68(61-75)$ & $73(71-77)$ & 0.28 \\
\hline Gender, n (\%) & & & & 0.62 \\
\hline Male & $5(22 \%)$ & $2(9 \%)$ & $3(13 \%)$ & \\
\hline Female & $18(78 \%)$ & $5(22 \%)$ & $13(57 \%)$ & \\
\hline ECOG performance status, n (\%) & & & & $>0.99$ \\
\hline $0-1$ & $16(70 \%)$ & $5(22 \%)$ & $11(48 \%)$ & \\
\hline $2-3$ & $7(30 \%)$ & $2(9 \%)$ & $5(22 \%)$ & \\
\hline Smoking history, $\mathrm{n}(\%)$ & & & & 0.32 \\
\hline Never smokers & $17(74 \%)$ & $4(17 \%)$ & $13(57 \%)$ & \\
\hline Former smokers & $6(26 \%)$ & $3(13 \%)$ & $3(13 \%)$ & \\
\hline Histology, n (\%) & & & & - \\
\hline Adenocarcinoma & $23(100 \%)$ & $7(30 \%)$ & $16(70 \%)$ & \\
\hline$E G F R$ mutation type at diagnosis, $\mathrm{n}(\%)$ & & & & $0.65^{*}$ \\
\hline Exon 19 deletion & $12(52 \%)$ & $4(17 \%)$ & $8(35 \%)$ & \\
\hline Exon $21 \mathrm{~L} 858 \mathrm{R}$ & $10(44 \%)$ & $2(9 \%)$ & $8(35 \%)$ & \\
\hline Exon 18 G719S* & $1(4 \%)$ & $1(4 \%)$ & $0(0 \%)$ & \\
\hline Rebiopsy, n (\%) & & & & $>0.99$ \\
\hline Tissue & $21(91 \%)$ & $7(30 \%)$ & $14(60 \%)$ & \\
\hline Liquid & $2(9 \%)$ & $0(0 \%)$ & $2(9 \%)$ & \\
\hline No. of prior therapies, median (range) & $4(2-5)$ & $4(2-4)$ & $4(2-5)$ & 0.39 \\
\hline \multicolumn{5}{|l|}{ Metastases, $\mathrm{n}(\%)$} \\
\hline Pleural effusion & $7(30 \%)$ & $7(30 \%)$ & $0(0 \%)$ & - \\
\hline Brain & $10(43 \%)$ & 4 & 6 & 0.65 \\
\hline Bone & $11(48 \%)$ & 3 & 8 & $>0.99$ \\
\hline Liver & $3(13 \%)$ & 1 & 2 & $>0.99$ \\
\hline
\end{tabular}

ECOG, Eastern Cooperative Oncology Group; EGFR, epidermal growth factor receptor. *Exon 18 G719S was excluded from analysis because only one patient had this mutation.

was evaluated using the Response Evaluation Criteria in Solid Tumors (RECIST) version 1.1 (7). All patients were confirmed to have the EGFR T790M mutation in tissue or blood samples using the Cobas ${ }^{\circledR}$ EGFR Mutation Test, version 2 (Roche Molecular Diagnostics, Pleasanton, CA, USA).

Statistical analysis. TTF and OS were determined using the KaplanMeier method, and comparisons between subgroups were performed using a log-rank test. $p$-Values less than 0.05 were considered significant. Hazard ratios were calculated using the Cox proportional hazards models. All statistical analyses were performed using EZR version 1.27 (Saitama Medical Center, Jichi Medical University, Saitama, Japan), which is a graphical user interface for R (The R Foundation for Statistical Computing, Vienna, Austria).

\section{Results}

Patients. Twenty-three patients were treated with osimertinib during the period covered by this study; their baseline characteristics are summarized in Table I. The median age at the time of osimertinib treatment was 73 years (range $=43-87$ years); $78 \%$ of the patients were women, while $74 \%$ were never smokers. Moreover, $30 \%$ of the patients had an Eastern Cooperative Oncology Group (ECOG) performance status score of 2 or higher. All patients had adenocarcinoma histology, and EGFR mutations including exon 19 deletion, L858R, and exon 18 G719S were detected before administering first or secondgeneration EGFR-TKIs. Disease in approximately $90 \%$ of patients was diagnosed using repeat tissue biopsies. A total of $30 \%$ of patients had pleural effusions, while $43 \%, 48 \%$, and $13 \%$ had metastatic lesions in the brain, bone, and liver, respectively. Patients were categorized into two groups based on whether pleural effusion was present or not; the characteristics of patients in these groups were not significantly different (Table I).

Efficacy. The median TTF of the whole patient cohort was 11.6 months $[95 \%$ confidence interval $(\mathrm{CI})=3.75$ monthsnot attained (NA)] (Figure 1A), while the median OS was 17.8 months (95\% CI=10.9 months-NA) (Figure 1B). Patients with pleural effusions had a significantly shorter median TTF than those without (3.7 vs. 12.8 months, respectively, $p=0.021$ ) (Figure $2 \mathrm{~A}$ ). Similarly, patients with pleural effusions had a significantly shorter median OS than those without (7.8 months vs. NA, respectively, $p=0.002$ ) (Figure 2B). On the other hand, the presence of other metastases did not significantly influence the median 

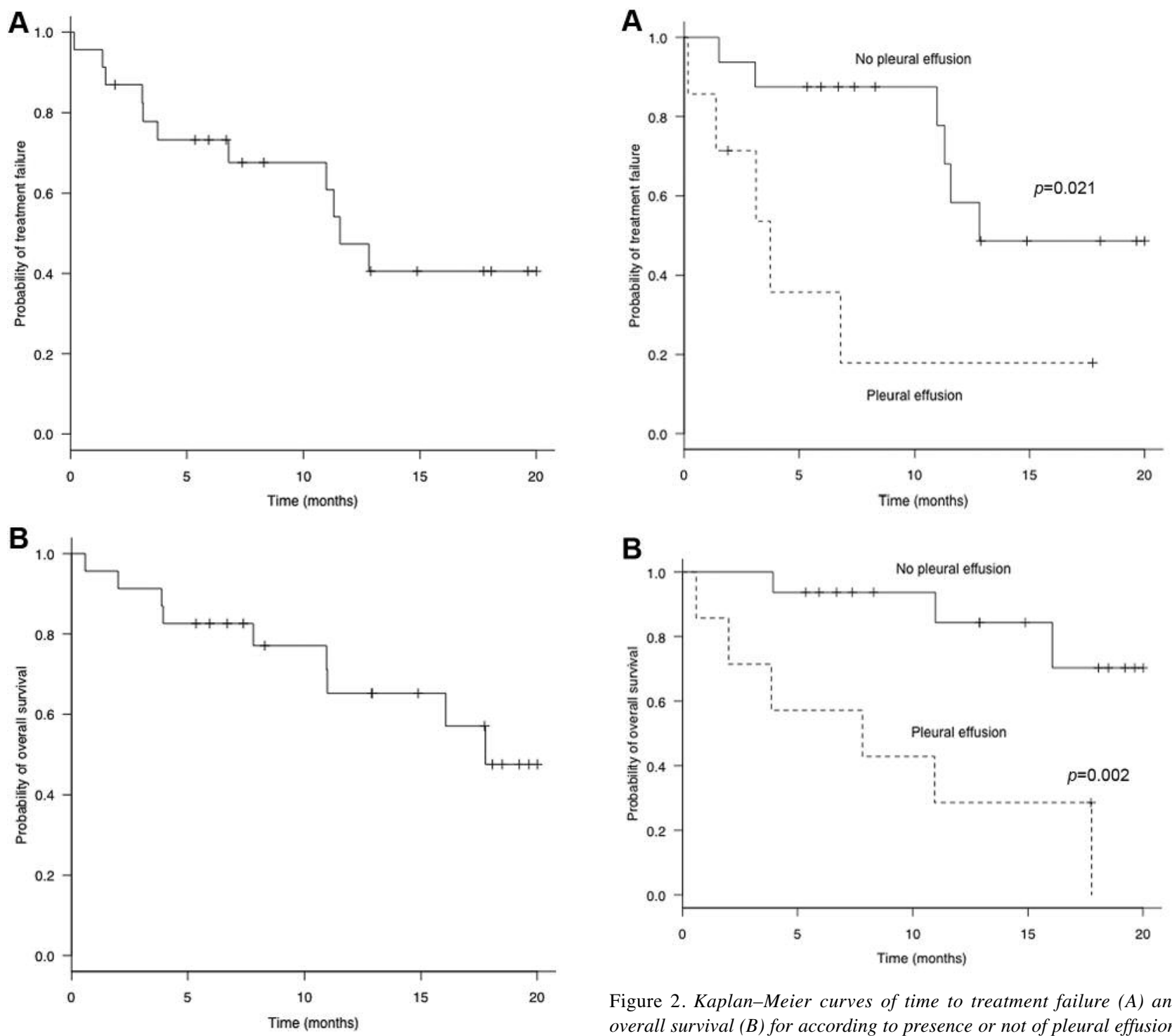

Figure 2. Kaplan-Meier curves of time to treatment failure (A) and overall survival $(B)$ for according to presence or not of pleural effusion. Median time to treatment failure was 3.7 (95\% CI=0.16-NA) vs. 12.8 (95\% CI=10.9-NA) months, respectively $(p=0.021)$. Median overall

Figure 1. Kaplan-Meier curves of time to treatment failure $(A)$ and overall survival $(B)$ in the whole patient cohort. Median time to treatment failure was 11.6 months [95\% confidence interval $(C I)=3.75$ months-not attained (NA)] (Figure 1A), while the median overall survival was 17.8 months (95\% CI=10.9 months-NA).

TTF (Table II). The response rate for the entire cohort was $56.5 \%$, while the disease control rate was $82.6 \%$. In subgroup analysis, the response rate in patients with pleural effusion was $28.6 \%$, while that of patients without was $68.8 \%$; in comparison, the response rate of patients with brain metastases was $70.0 \%$ and that in patients without was $46.2 \%$. Only one out of the seven patients with pleural effusions experienced a reduction in effusion volume with treatment.

\section{Discussion}

In this retrospective study, we found that patients with pleural effusions upon initiating osimertinib treatment had significantly shorter TTF and OS than those without effusion. In contrast, there were no significant differences in these durations based on the presence of brain, bone, and liver metastases. Our data suggest that osimertinib is less effective in patients with pleural effusion than in those with distant metastasis to other organs. 
Table II. Prognostic factors associated with time to treatment failure.

\begin{tabular}{lccc}
\hline Factor & \multicolumn{3}{c}{ Time to treatment failure (months) } \\
\cline { 2 - 4 } & HR & $95 \%$ CI & $p$-Value \\
\hline Age $\geq 75$ years & 1.36 & $0.41-4.5$ & 0.61 \\
Female & 1.87 & $0.24-14.7$ & 0.55 \\
Exon 21 L858R* & 2.16 & $0.60-7.7$ & 0.24 \\
ECOG performance status $\geq 2$ & 1.06 & $0.28-4.0$ & 0.93 \\
Pleural effusion & 3.81 & $1.1-12.8$ & 0.021 \\
Brain metastases & 1.84 & $0.5-6.3$ & 0.33 \\
Bone metastases & 1.29 & $0.4-4.2$ & 0.67 \\
Liver metastases & 2.63 & $0.7-10.0$ & 0.14 \\
\hline
\end{tabular}

ECOG, Eastern Cooperative Oncology Group; HR, hazard ratio; CI, confidence interval. *Exon 18 G719S was excluded as only one patient had this mutation.

Pleural effusions occur as a consequence of tumor cell invasion into the pulmonary artery, which causes embolization of the pulmonary pleura;they can also be seeded by peripheral tumors (8). The existence of pleural effusion is reportedly an independent predictor of poor survival in patients with advanced NSCLC (9). In this study, we found that presentation with pleural effusion at baseline resultsed in significantly shorter TTF and OS in patients treated with osimertinib. This indicates that effectiveness of this agent is reduced in patients with NSCLC with pleural effusions at baseline, which was also shown to be the case in those treated with first-and second-generation EGFR-TKIs $(5,6)$. While the reason for this reduced effectiveness is not clear, it was shown that the expression of vascular endothelial growth factor (VEGF), a critical cytokine in pleural effusion pathogenesis, is associated with resistance to EFGR-TKIs $(10,11)$. Therefore, osimertinib likely has the same limitations as its predecessors in this regard. In patients who develop malignant pleural effusion after acquiring resistance to previous-generation EGFR-TKIs, adding the VEGF inhibitor bevacizumab to their regimens reportedly prolongs their PFS (12).

Our study found no differences in survival with osimertinib treatment when comparing patients with $v s$. without NSCLC metastases to the brain, bone, or liver. Previous studies of the efficacies of first- or secondgeneration EGFR-TKIs in patients with different sites of metastasis at baseline found that brain, bone, and liver metastases were independent poor predictors of PFS and OS (13-16). Although this discrepancy may be due to the small sample size of our cohort, it remains unclear whether this is a result of differences between EGFR-TKIs. Conversely, a subset of the AURA 3 trial showed that treatment with osimertinib was associated with a median PFS of 8.5 months in patients with brain metastases of NSCLC, while platinum- pemetrexed treatment produced a median PFS of 4.2 months (1). The BLOOM trial also reported 21 patients with leptomeningeal metastases treated with osimertinib (160 mg orally, once daily) who achieved a $43 \%$ response rate (17). Although their number was small, the response rate of patients with brain metastases in our study was high at $70.0 \%$. These data indicate that osimertinib may be more effective in patients with brain metastases than previous generation EGFR-TKIs.

Our study had several limitations. Firstly, we retrospectively analyzed data from a single institution, and our sample size was too small to lead to definite conclusions. Secondly, our study included patients who experienced PD, but were nevertheless deemed to derive some benefit from continuing osimertinib.

In conclusion, osimertinib is effective in patients with EGFR T790M-positive advanced NSCLC. However, similarly to first- and second-generation EGFR-TKIs, osimertinib monotherapy appears to be less effective in patients with NSCLC with pleural effusions. Further prospective studies are warranted to confirm these findings.

\section{Conflicts of Interest}

The Authors have no conflicts of interest to declare in regard to this study.

\section{References}

1 Mok TS, Wu Y-L, Ahn M-J, Garassino MC, Kim HR, Ramalingam SS, Shepherd FA, He Y, Akamatsu H, Theelen WS, Lee CK, Sebastian M, Templeton A, Mann H, Matotti M, Ghiorghiu S and Papadimitrakopoulou VA; AURA3 Investigators: Osimertinib or platinum-pemetrexed in EGFR T790M-positive lung cancer. N Engl J Med 376: 629-640, 2017.

2 Soria JC, Ohe Y, Vansteenkiste J, Reungwetwattana T, Chewaskulyong B, Lee KH, Dechaphunkul A, Imamura F, Nogami N, Kurata T, Okamoto I, Zhou C, Cho BC, Cheng Y, Cho EK, Voon PJ, Planchard D, Su WC, Gray JE, Lee SM, Hodge R, Marotti M, Rukazenkov Y and Ramalingam SS; FLAURA Investigators: Osimertinib in untreated EGFR-mutated advanced non-small-cell lung cancer. N Engl J Med 378: 113$125,2018$.

3 Zou J, Bella AE, Chen Z, Han X, Su C, Lei Y and Luo H: Frequency of EGFR mutations in lung adenocarcinoma with malignant pleural effusion: Implication of cancer biological behavior regulated by EGFR mutation. J Int Med Res 42: 1110$1117,2014$.

4 Tsai MF, Chang TH, Wu SG, Yang HY, Hsu YC, Yang PC and Shih JY: EGFR-L858R mutant enhances lung adenocarcinoma cell invasive ability and promotes malignant pleural effusion formation through activation of the CXCL12-CXCR4 pathway. Sci Rep 5: 13574, 2015.

5 Atagi S, Goto K, Seto T, Yamamoto N, Tamura T, Tajima K and Inagaki N: Erlotinib for Japanese patients with activating $E G F R$ mutation-positive non-small-cell lung cancer: combined analyses from two phase II studies. Future Oncol 12: 2117-2126, 2016. 
6 Taniguchi Y, Tamiya A, Nakahama K, Naoki Y, Kanazu M, Omachi N, Okishio K, Kasai T and Atagi S: Impact of metastatic status on the prognosis of EGFR mutation-positive non-small cell lung cancer patients treated with first-generation $E G F R$ tyrosine kinase inhibitors. Oncol Lett 14: 7589-7596, 2017.

7 Eisenhauer EA, Therasse P, Bogaerts J, Schwartz LH, Sargent D, Ford R, Dancey J, Arbuck S, Gwyther S, Mooney M, Rubinstein L, Shankar L, Dodd L, Kaplan R, Lacombe D and Verweij J: New response evaluation criteria in solid tumours: revised RECIST guideline (version 1.1). Eur J Cancer 45: 228247, 2009.

8 Lynch TJ Jr.: Management of malignant pleural effusions. Chest 103: 385S-389S, 1993.

9 Morgensztern D, Waqar S, Subramanian J, Trinkaus K and Govindan R: Prognostic impact of malignant pleural effusion at presentation in patients with metastatic non-small-cell lung cancer. J Thorac Oncol 7: 1485-1489, 2012.

10 Economidou F, Margaritopoulos G, Antoniou KM and Siafakas NM: The angiogenetic pathway in malignant pleural effusions: Pathogenetic and therapeutic implications. Exp Ther Med 1: 3 7, 2010

11 Naumov GN, Nilsson MB, Cascone T, Briggs A, Straume O, Akslen LA, Lifshits E, Byers LA, Xu L, Wu HK, Jänne P, Kobayashi S, Halmos B, Tenen D, Tang XM, Engelman J, Yeap B, Folkman J, Johnson BE and Heymach JV: Combined vascular endothelial growth factor receptor and epidermal growth factor receptor $(E G F R)$ blockade inhibits tumor growth in xenograft models of EGFR inhibitor resistance. Clin Cancer Res 15: 34843494, 2009.

12 Jiang T, Li A, Su C, Li X, Zhao C, Ren S, Zhou C and Zhang J: Addition of bevacizumab for malignant pleural effusion as the manifestation of acquired EGFR-TKI resistance in NSCLC patients. Oncotarget 8: 62648-62657, 2017.
$13 \mathrm{Wu}$ KL, Tsai MJ, Yang CJ, Chang WA, Hung JY, Yen CJ, Shen $\mathrm{CH}$, Kuo TY, Lee JY, Chou SH, Liu TC, Chong IW and Huang MS: Liver metastasis predicts poorer prognosis in stage IV lung adenocarcinoma patients receiving first-line gefitinib. Lung Cancer 88: 187-194, 2015.

14 Fujimoto D, Ueda H, Shimizu R, Kato R, Otoshi T, Kawamura T, Tamai K, Shibata Y, Matsumoto T, Nagata K, Otsuka K, Nakagawa A, Otsuka K, Katakami N and Tomii K: Features and prognostic impact of distant metastasis in patients with stage IV lung adenocarcinoma harboring EGFR mutations: importance of bone metastasis. Clin Exp Metastasis 31: 543-551, 2014.

15 Liang SK, Hsieh MS, Lee MR, Keng LT, Ko JC and Shih JY: Real-world experience of afatinib as a first-line therapy for advanced EGFR mutation-positive lung adenocarcinoma. Oncotarget 8: 90430-90443, 2017.

16 Lin JH, Lin D, Xu L, Wang Q, Hu HH, Xu HP and He ZY: The association between clinical prognostic factors and epidermal growth factor receptor-tyrosine kinase inhibitor (EGFR-TKI) efficacy in advanced non-small-cell lung cancer patients: a retrospective assessment of 94 cases with EGFR mutations. Oncotarget 8: 3412-3421, 2017.

17 Yang JC, Cho BC, Kim DW, Kim SW, Lee JS and Su WC: Osimertinib for patients with leptomeningeal metastases from EGFR-mutant non-small cell lung cancer: Updated results from the BLOOM study. J Clin Oncol 35(Suppl 15): 2020, 2017.

Received April 3, 2018

Revised May 8, 2018

Accepted May 9, 2018 